\title{
ABORDAGEM CONTRASTIVA NA TERAPIA DOS DESVIOS FONOLÓGICOS: CONSIDERAÇÕES TEÓRICAS
}

\author{
Contrastive approach in phonological disorders therapy: \\ theoretical considerations
}

Karina Carlesso Pagliarin ${ }^{(1)}$, Márcia Keske-Soares ${ }^{(2)}$

\begin{abstract}
RESUMO
Objetivo: realizar uma revisão de literatura sobre os modelos terapêuticos utilizados na terapia fonoaudiológica, dando-se ênfase aos modelos com abordagem contrastiva, sendo estes o de Pares Mínimos/Oposições Máximas e o de Oposições Múltiplas. Métodos: foram realizadas buscas a partir de pesquisa de publicações nas bases de dados Medline, Scielo, Bireme e Pubmed, sendo também utilizados livros, teses, dissertações, monografias e artigos de periódicos. Resultados: os modelos de Pares Mínimos/Oposições Máximas envolvem a seleção de pares de palavras que se diferenciam por um único fonema, podendo contrastar poucos traços distintivos (pares mínimos) ou muitos traços (oposições máximas). Esses modelos são preferencialmente indicados para crianças com desvio médio a médio-moderado. No modelo de Oposições Múltiplas, são selecionadas palavras que também diferem em apenas um fonema, porém estes contrastam diversos traços devido à seleção de vários fonemas. Esse modelo é recomendado para desvio severo, pois, em geral, as crianças substituem muitos fonemas do sistema adulto por um único som. Conclusão: para a escolha do modelo adequado, devem-se levar em consideração a presença e a ausência dos fonemas no sistema fonológico da criança, o tipo e a gravidade do desvio fonológico. Além disso, a seleção do modelo terapêutico adequado pode auxiliar nas generalizações a serem obtidas.
\end{abstract}

DESCRITORES: Transtornos da Articulação; Fala; Fonoterapia

\section{INTRODUÇÃO}

Os desvios fonológicos são alterações de fala que se caracterizam pelo apagamento, substituições, inserções e/ou reordenamentos de sons no sistema fonológico da criança, fazendo com que a fala tornese incompreensível para o ouvinte ${ }^{1,2}$.

Os modelos de terapia com base fonológica têm proposta baseada na generalização, ou seja, prevê que um som trabalhado é utilizado corretamente em ou-

(1) Fonoaudióloga; Bolsista CAPES do Programa de Pós-Graduação em Distúrbios da Comunicação Humana; Especialização em Fonoaudiologia pela Universidade Federal de Santa Maria; Mestranda em Distúrbios da Comunicação Humana pela Universidade Federal de Santa Maria.

(2) Fonoaudióloga; Professora do Curso de Fonoaudiologia e do Mestrado em Distúrbios da Comunicação Humana da Universidade Federal de Santa Maria; Doutora em Lingüística Aplicada pela Pontifícia Universidade Católica do Rio Grande do Sul. tros ambientes e/ou sons não treinados, diminuindo, deste modo, o tempo de tratamento de uma criança com desvio fonológico. Para isso, o fonoaudiólogo deve ter em mente o modelo terapêutico e seus princípios teóricos, realizar as avaliações e selecionar sons-alvo adequados ao tratamento, os quais devem possibilitar a ocorrência de generalizações ${ }^{3}$.

O tratamento dos desvios fonológicos tem sido muito estudado principalmente quanto aos modelos terapêuticos e seus princípios teóricos ${ }^{4-11}$.

Os modelos devem ser utilizados de acordo com o tipo de sistema fonológico apresentado pela criança ${ }^{12}$. Existem diversos modelos para o tratamento dos desvios (Metaphon ${ }^{13}$, Ciclos Modificado ${ }^{14}$, Modelo "ABAB - Retirada e Provas Múltiplas" ${ }^{15}$ ) e alguns utilizam a abordagem contrastiva de traços distintivos para a escolha dos sons-alvo, dentre estes se destacam o Modelo de Pares Mínimos ${ }^{16}$, o de Oposições Máximas ${ }^{17}$ e o de Oposições Máximas Modificado ${ }^{11}$, aplicados em crianças falantes do Português Brasileiro (PB) em algumas pesquisas ${ }^{6,18-20}$ 
no Brasil. Outro modelo de oposições, o Modelo de Oposições Múltiplas ${ }^{4}$ ainda é pouco estudado e aplicado em crianças falantes do PB.

Este estudo teve por objetivo apresentar uma revisão de literatura sobre os modelos de oposições contrastivas (Modelo de Pares Mínimos/Oposições Máximas e Oposições Múltiplas).

\section{MÉTODOS}

Realizou-se uma revisão sistemática da literatura através de pesquisa em diferentes bases de dados como MEDLINE, SCIELO, BIREME e PUBMED, no período de março a agosto de 2006, utilizando as seguintes palavras-chave: "distúrbio da fala", "speech disorders", "fonoterapia", "speech therapy", "transtornos da articulação", "articulation disorders", "Gierut, J. A." e "Williams A. L.". Nesta revisão, foram encontrados 522 trabalhos publicados, dos quais, após leitura e análise, 44 foram utilizados neste estudo. A análise dos resultados foi realizada mediante a comparação quanto à aplicação e os resultados obtidos a partir do tratamento com os modelos terapêuticos com abordagem contrastiva, descritos na literatura. Além da busca em base de dados, nesta pesquisa, foram usados também livros, teses, dissertações e monografias.

\section{RESULTADOS}

Alguns autores ${ }^{21,22}$ definem o desvio fonológico como uma dificuldade no domínio da fonologia. Contudo, o termo permite a possibilidade de imprecisões articulatórias, além dos problemas na organização do sistema de sons ${ }^{22}$.

Entre o nascimento e a idade de cinco anos, na maioria das crianças, ocorre o amadurecimento do conhecimento fonológico num processo gradativo, não linear e com variações individuais, resultando no estabelecimento de um sistema condizente com o alvoadulto. No entanto, existem crianças em que a maneira como o seu sistema fonológico é construído difere quanto ao caminho percorrido, sendo o mesmo diferente da fonologia da língua do seu ambiente e, portanto, inadequado em relação a esta, sendo denominadas de crianças com desvios fonológicos ${ }^{23}$.

Existem diferentes propostas para a classificação dos desvios fonológicos, sendo que algumas utilizam abordagens qualitativas ${ }^{24-27}$, que se baseiam na inteligibilidade da fala e na gravidade, enquanto que outras ${ }^{27-29}$ fazem uma análise predominantemente quantitativa, sendo esta última bastante utilizada, tanto em pesquisas nacionais como internacionais. Um exemplo desse tipo de análise é o Percentual de Consoantes Corretas (PCC) ${ }^{30}$, o qual é obtido mediante a divisão do número de consoantes corretas pelo número total de consoantes, isto é, corretas mais incorretas, após o resultado é multiplicado por cem. Com o resultado do PCC, o desvio pode ser classificado como desvio médio (86 a 100\%); desvio médio moderado (66 a 85\%); desvio moderado-grave (51 a $65 \%)$; e desvio grave (<50\%). Esta é uma das análises mais utilizadas em pesquisas que envolvem o desvio fonológico, muitas vezes realizado juntamente com outros parâmetros, como o PDI (Process Density Index) ${ }^{31}$.

A avaliação é uma das principais ferramentas do terapeuta da fala-linguagem, pois ela pode acelerar o processo terapêutico. Uma avaliação bem realizada leva a um diagnóstico bem determinado, à identificação da etiologia e de condições agravantes, fornecendo uma base para a intervenção ${ }^{12}$

A terapia para o desvio fonológico deve enfatizar a reorganização do sistema de sons da criança, tendo como base o sistema padrão-adulto. A incorporação de novos fonemas no sistema fonológico é realizada através do conhecimento fonológico adquirido no tratamento, em que não são ressaltados exercícios motores, sendo estes apenas um auxílio, quando necessário ${ }^{27}$.

A terapia dos desvios da fala baseava-se, antes das abordagens lingüísticas atuais, exclusivamente na produção fonética. Os fonemas eram tratados de forma isolada, depois em sílabas, palavras e frases, finalizando com a fala espontânea. A mudança para o novo alvo só se dava após a automatização do primeiro. Os tratamentos, dessa maneira, eram demasiadamente longos ${ }^{22}$.

O que diferencia um modelo de terapia tradicional de um modelo baseado na fonologia é que a escolha dos processos e dos sons-alvo, no tratamento, baseia-se em hipóteses sobre a estrutura organizacional do sistema fonológico da criança ${ }^{22}$.

$\mathrm{Na}$ abordagem terapêutica atual, com base fonológica, a generalização é o critério mais importante para se medir o sucesso obtido com o tratamento, sendo esta a ampliação da produção e uso correto de fones-alvo estimulados em terapia para outros contextos ou ambientes não trabalhados. A generalização pode ser de dois tipos: estrutural e funcional. $O$ primeiro refere-se à identificação das propriedades estruturais da generalização ou circunstâncias sob as quais ela ocorre, enquanto que o segundo tipo examina as propriedades funcionais da generalização ou como esta é usada por uma criança para modificar seu sistema fonológico, analisando as variáveis intra-sujeitos, que podem ser a maturidade lingüística, o funcionamento cognitivo e as habilidades motoras da criança ${ }^{32}$.

Os principais tipos de generalização estrutural são: a itens (palavras) não utilizados no tratamento; para outra posição da palavra; para outras unidades lingüísticas; dentro de uma classe de sons; para outras classes de sons; para outras situações; e 
baseada nas relações implicacionais ${ }^{32}$. Estes diversos tipos foram bastante estudados ${ }^{6,9,10,19,29}$ e apresentados os resultados obtidos em diferentes modelos de terapia com base fonológica.

Há diversos modelos de terapia com base fonológica, os quais se diferenciam segundo a teoria fonológica que os fundamenta, os princípios terapêuticos e o modo como são aplicados. Dentre os mais utilizados, principalmente no sul do Brasil, estão os seguintes modelos terapêuticos: Metaphon ${ }^{13}$, aplicado em crianças falantes do português ${ }^{33}$; Ciclos Modificado ${ }^{14}$, aplicado em crianças falantes do português ${ }^{34}$; Modelo de Pares Mínimos/Oposições Máximas ${ }^{35}$, aplicado em crianças falantes do português ${ }^{18}$; e o Modelo "ABAB Retirada e Provas Múltiplas" ${ }^{15}$, aplicado em crianças falantes do português ${ }^{27}$. O Modelo de Oposições Múltiplas ${ }^{4}$ trata-se de um modelo que pressupõe a abordagem contrastiva, no entanto, não foi ainda aplicado em crianças falantes do português.

A seguir serão detalhados os modelos de abordagem contrastiva, foco desta revisão teórica (Modelo de Pares Mínimos/Oposições Máximas e Modelo de Oposições Múltiplas).

No método de terapia com contraste mínimo ${ }^{36}$ (pares mínimos), muito utilizado atualmente, são selecionados pares de palavras que se distinguem por uma única consoante ou vogal, mas que são produzidas como homônimos pela criança. A intervenção envolve a seleção de pares de palavras que contrastam a produção errada da criança com o som-alvo, comparando um com outro.

Um estudo ${ }^{16}$ investigou o contraste mínimo ensinando oposições fonológicas a duas crianças, falantes do inglês, com idades de 4:10 e 4:4, com fala ininteligível. Nesse método de tratamento, foram selecionados pares mínimos os quais a criança, devido a seu padrão desviante, pronunciaria o primeiro membro do par, chamado palavra-alvo, exatamente da mesma maneira que o segundo membro. Por exemplo: quando uma criança substitui a plosiva /b/ por [p], no momento em que ela emite a palavra "bato" ['bato] ela produz ['pato], não havendo, dessa maneira, uma distinção. Com isso é possível mostrar à criança que sua alteração de fala resulta em problemas de comunicação. O modelo foi efetivo no tratamento dos sujeitos estudados, sendo que os mesmos apresentaram diminuição de processos fonológicos e houve generalização para palavras não tratadas e para outras classes de sons.

O tratamento com pares mínimos ${ }^{37}$ consiste na seleção de dois sons-alvo, os quais diferem quanto aos traços distintivos. Com esses sons, são selecionados pares de palavras sem sentido (nonsense words-NSW), onde o significado das mesmas é determinado dentro do contexto de estórias. As palavras sem sentido são ensinadas usando uma variedade de atividades basea- das conceitualmente: escolha, competição, contar histórias informalmente, entre outros. O tratamento é realizado em duas fases: produção espontânea e imitação. Em ambas as fases, a criança é apresentada a pares de desenhos de palavras sem sentido. Na fase imitativa, a criança repete o modelo verbal do terapeuta. O tratamento continua até a criança manter $75 \%$ de produção imitativa correta em duas sessões consecutivas dentro de uma forma de par mínimo ou sete sessões consecutivas. $\mathrm{O}$ tratamento então passa para a fase espontânea com a produção da criança das palavras sem o modelo do terapeuta. Esta fase continua até a criança manter uma produção correta de $90 \% \mathrm{em}$ três sessões consecutivas com uma forma de par mínimo ou doze sessões consecutivas. As crianças devem ser verbalmente elogiadas nas respostas corretas, seguindo um programa contínuo durante a fase imitativa e um programa intermitente durante a fase espontânea. Se uma resposta for julgada incorreta, o terapeuta apresenta o modelo verbal da palavra sem sentido para a criança imitar.

Esta proposta de tratamento com pares mínimos ${ }^{17,37}$ envolve pares de duas palavras que diferem em apenas um fonema. Se estes fonemas diferem em poucos traços distintivos formam as oposições mínimas, e em vários traços, as oposições máximas. A maneira como os fonemas de um par mínimo difere pode ser fundamental na indução de mudanças fonológicas na terapia. Os fonemas de um par mínimo podem diferir em três dimensões:

- em relação ao número de oposições distintivas, isto é, o número de traços específicos em que os fonemas diferem os mesmos podem ser minimamente opostos (diferindo por poucos traços) ou maximamente opostos (diferindo por muitos traços);

- em relação à natureza dos traços distintivos, ou seja, os fonemas podem diferir em traços de classe principal ou classe não principal. Os traços de classe principal são aqueles que descrevem e distinguem as vogais, líquidas e nasais, obstruintes e glides. As distinções de classes principais referem-se às diferenças entre os fonemas envolvendo os traços [soante], [consonantal] e/ou [silábico], de acordo com a matriz de traços ${ }^{38}$. Classes distintivas não principais referem-se a todas as outras diferenças de ponto, modo e sonoridade entre os fonemas.

- quanto à sua relação com a gramática da criança pré-tratamento: o fonema tratado pode ser desconhecido ou não funcional (ausente) no sistema fonológico da criança, ou conhecido, funcional (presente) em seu sistema fonológico.

Outra pesquisa ${ }^{17}$ analisou se o número de traços distintivos, classes de traços e as relações dos fonemas tratados com a gramática da criança antes do tratamento influenciavam nas mudanças dos sistemas fonológicos em crianças com desvios 
fonológicos. Foram estudados quatro sujeitos, três meninos e uma menina, com idade média de 3:10, falantes do inglês. Cada sujeito foi exposto a duas formas diferentes de tratamentos com pares mínimos. O par mínimo tratado consistiu em dois fonemas ausentes no sistema fonológico da criança pré-tratamento e o par alternado em um fonema ausente, comparado com um fonema conhecido para a criança, isto é, presente em seu sistema fonológico. Todos os pares diferiam em número máximo de oposições fonológicas (traços), e eram diferenciados pela natureza das oposições fonológicas, ou seja, distinções de classe principais ou de classes não principais. $O$ tratamento apresentou todas as combinações lógicas possíveis: classes de traços e relação dos fonemas tratados com a gramática da criança.

Os pares mínimos eram apresentados através de palavras sem sentido. As sessões de terapia ocorreram três vezes por semana, com duração de 60 minutos. Para avaliar as mudanças ocorridas nos sistemas fonológicos das crianças, foram realizadas provas de generalização. Após a análise dos resultados, foi elaborada uma hierarquia de mudanças fonológicas a partir da aplicação dos diferentes formatos de tratamento, a qual está apresentada na Figura 1. De acordo com esta hierarquia, o tratamento de pares mínimos que introduz dois fonemas novos, com distinções máximas de traços e distinções de traços de classe principal, conduz a maiores mudanças no sistema fonológico da criança. O formato que combina um fonema novo e outro conhecido, com diferenças máximas de traços e diferença de traços de classe principal e o formato que combina dois fonemas novos, com distinções máximas de traços e traços com diferença de classe não principal, leva a mudanças fonológicas intermediárias. O tratamento envolvendo um fonema novo, com distinções máximas de traços e diferença de traços de classe não principal, resulta em menores mudanças fonológicas ${ }^{17}$.

Pesquisas ${ }^{8,9,17-19,37,39}$ que testaram, no tratamento com pares mínimos, distinções mínimas e máximas de traços concluíram que, de fato, pares de sons maximamente opostos resultam em maiores generalizações do que pares minimamente opostos.

A generalização foi observada ${ }^{40}$, com base nas relações implicacionais, de quatro crianças, com idades entre 5:3 a 7:5, com desvio fonológico médio-moderado, as quais foram tratadas através do Modelo de Oposições Máximas. Dos quatro tipos de generalização analisados (a itens não utilizados no tratamento; para outra posição na palavra; dentro de uma classe de sons; e para outras classes de sons), as quatro crianças apresentaram todos os tipos de generalizações e expandiram seus sistemas fonológicos.

Um estudo ${ }^{41}$ realizado com o objetivo de comparar as mudanças fonológicas obtidas pelo tratamento com diferentes modelos de terapia em sujeitos com diferentes graus de severidade do desvio fonológico (DF), verificou que o Modelo de Oposições Máximas é eficaz principalmente em crianças que apresentam desvio moderado-grave, sendo observado também a ocorrência de diversas generalizações, como por exemplo: a itens não utilizados no tratamento, a outras posições na palavra, dentro de uma classe de sons e para outras classes de sons.

O Modelo de Oposições Máximas Modificado ${ }^{11}$ foi uma proposta de terapia modificada com base no Modelo de Oposições Máximas ${ }^{35}$, tendo sido introduzidos procedimentos relacionados à escolha dos sons-alvo, à terapia fonológica e à estrutura da sessão. Assim, conforme os procedimentos utilizados na realização de outros modelos de terapia fonológica, foi proposta a realização de bombardeio auditivo, orientação aos pais, uso de palavras-alvo com significado e o cuidado na seleção das mesmas. A introdução de procedimentos mostrou-se efetiva na aplicação do modelo, o qual foi aplicado em um sujeito de 6:4, com desvio fonológico, falante do Português Brasileiro, durante 25 sessões terapêuticas.

O Modelo de Oposições Máximas Modificado possui o mesmo objetivo do modelo original ${ }^{17}$, isto é, de promover a reorganização do sistema fonológico da criança por meio da percepção auditiva, imitação da produção e produção espontânea dos sons-alvo, visando à generalização e a melhora na inteligibilidade da fala da criança.

Neste modelo, assim como no modelo original, para a escolha dos sons-alvo de tratamento, é necessário analisar o sistema fonológico da criança e, a partir das mudanças fonológicas, devem ser escoIhidos os sons-alvo, preferencialmente selecionar os sons-alvo que contenham os traços distintivos que a criança apresenta dificuldade. Após, serão selecionados os pares mínimos que irão contrastar estes sons (dois a quatro pares).

Com relação aos procedimentos terapêuticos, é realizada, inicialmente, a linha de base, ou seja, uma sondagem realizada antes do início da terapia onde cada som não-adquirido é testado, selecionando-se no máximo seis palavras que contenham este fonema e possam ser representadas por figuras, as quais a criança deve nomeá-las sem o modelo do terapeuta. A linha de base é realizada a fim de se ter um parâmetro inicial para comparar os progressos do tratamento e as generalizações ocorridas durante o mesmo.

Após a determinação da linha de base, inicia-se o tratamento propriamente dito. No modelo modificado, são realizadas cinco sessões de estimulação com os pares selecionados; na sexta sessão, realiza-se a primeira sondagem, a qual será efetuada da mesma maneira que a linha de base; repetem-se mais cinco sessões com os mesmos pares míni- 
mos; e novamente na sexta sessão, realizar outra sondagem. Continuar a terapia realizando sempre cinco sessões e uma sondagem; após 20 a 25 sessões de terapia (sem contar as sondagens), realizar novamente uma avaliação do sistema fonológico. A sessão terapêutica deve ser iniciada e terminada com o bombardeio auditivo, que consiste em uma lista de palavras que será lida para a criança e esta deve apenas ouvir, sem necessidade de repetir. A lista é formada por 16 palavras, das quais oito precisam conter um som-alvo e as oito restantes devem conter o outro som-alvo que formam os pares mínimos em questão, na mesma posição da palavra em que os sons-alvo são apresentados nos pares mínimos.

O Modelo de Oposições Múltiplas ${ }^{4}$ recorre diretamente aos múltiplos apagamentos de sons do sistema adulto, que resulta em substituições extensivas dos fonemas. Quando a função contrastiva de vários fonemas está ausente, o resultado é a presença de homônimos, isto é, quando duas ou mais palavras são produzidas de modo semelhante, mas possuem significados diferentes. Por exemplo: uma criança que substitui obstruintes surdas, os fonemas $/ \mathrm{s}, \mathrm{s}, \mathrm{k}, \mathrm{t} / \mathrm{r}$ realiza como [t], produziria as palavras sip, ship, kip, e chip como [tip]. Embora os significados e a pronúncia sejam diferentes da do adulto, a criança produz todas as palavras como homônimos. Esta anormalidade resulta em redução da inteligibilidade e quebras de comunicação ${ }^{4}$.

Nesse modelo, a criança é confrontada com vários sons simultaneamente, considerando-se, também, o fonema substituído. Ameta é induzir divisões fonêmicas múltiplas que têm sido previamente desativadas a fim de reduzir os homônimos no sistema fonológico da criança. As oposições múltiplas são direcionadas às homonímias com o uso de pares contrastivos para um amplo conjunto de contrastes de traços que são diferentes daqueles usados com uma abordagem contrastiva simples de pares mínimos, por exemplo, pois essa, selecionaria apenas um som para ser contrastado e aprendido por vez.

A teoria de Oposições Múltiplas envolve o nível da perspectiva fonológica, que é som versus sistema. A perspectiva da oposição múltipla considera a organização sistemática de um sistema de som como fundamental. Baseado nessa suposição, a intervenção terá grande impacto quando (a) o sistema fonológico e organização da criança são descritos e (b) o aprendizado fonológico é sistematicamente indicado através da regra ou estratégia fonológica.

O Modelo de Oposições Múltiplas foi aplicado ${ }^{4}$ em uma criança com desvio fonológico grave, com idade de 3:5. A criança substituía as glides [w, y] e duas fricativas [s, $\delta$ ] pela líquida lateral /I/. Esses quatro fonemas substituídos diferenciavam-se pelo ponto, modo e sonoridade (líquida alveolar sonora, alveolar dessonorizada e fricativas palatal e glide lá- bio-velar). Baseada nessa produção, o tratamento foi iniciado com pares mínimos para remediar [s, $\left.\int, w\right]$ em posição inicial. Cada som-alvo foi treinado em cinco pares mínimos separados para cada um dos três sons-alvo, resultando em um total de 15 pares contrastivos. Uma prova de generalização foi elaborada para verificar o aprendizado dos sons-alvo em palavras não treinadas e foi administrada antes do tratamento, para estabelecer a linha de base e no final de cada três sessões de terapia para mensurar a generalização. A prova consistia de 10 itens para cada som-alvo e em cada posição tratada.

Após o início do tratamento com pares mínimos o desempenho da criança melhorou, entretanto após nove sessões, ou cinco semanas, a criança não apresentou progressos. Nesse ponto, o modelo de tratamento foi reavaliado para determinar quais mudanças poderiam ser feitas que facilitassem o aprendizado de [s, $\mathrm{s}$. A intervenção então, mudou do contraste de cada som-alvo em pares mínimos para o modelo de Oposições Múltiplas, que contrastou todos os sons-alvo com a substituição errada. Cinco grupos de oposições foram incorporados no tratamento desses fonemas substituídos. A criança demonstrou uma melhora significativa após a aplicação do modelo. Embora os resultados obtidos sejam limitados a um relato descritivo de uma criança cujo aprendizado fonológico foi facilitado pelo uso inicial do modelo de pares mínimos, os achados são intrigantes e interessantes no traçado da gênese clínica e teórica de um modelo contrastivo diferente e, ainda, sugerem que esta abordagem contrastiva fornece uma sistemática expansão do sistema fonológico na ausência de contrastes fonêmicos.

Uma pesquisa ${ }^{5}$ realizada com o objetivo de verificar a eficácia da terapia fonológica utilizando o modelo de oposições múltiplas apresenta os dados de intervenção fonológica em 10 crianças, sendo oito do sexo masculino e duas do sexo feminino (entre 4 e 5 anos de idade), com desvios fonológicos de grau moderado, grave e profundo. Os critérios de inclusão para o estudo foram: exclusão de pelo menos seis sons do inventário fonético; audição normal para fala; capacidades cognitivas normais; ausência de desordens orgânicas ou motoras; idade entre 42 e 78 meses; ser membro de uma família de falantes monolíngües do inglês. As crianças foram avaliadas antes e após a intervenção. Foram coletados dados de fala através de um teste de nomeação de 245 itens de palavras isoladas que continham todos os sons mínimos do inglês e em cada posição. Após, o sistema fonológico de cada criança foi descrito em termos de inventário fonético, processos fonológicos, análise contrastiva e contrastes fonotáticos.

O sistema fonológico das crianças foi caracterizado através do "correct underlying representations" 
(CUR) ou ambiente conhecido. A construção lingüística do CUR reflete a natureza dos morfemas no léxico mental da criança. De acordo com o mesmo as crianças são caracterizadas em três categorias de gravidade, baseadas na porcentagem de produção fonológica relativa ao sistema adulto padrão. A criança poderá ser classificada na categoria moderada se obter de $54 \%$ - $60 \%$ de acertos, na categoria grave de $37 \%$ - $43 \%$ e na categoria profunda de $14 \%-29 \%$.

Todas as crianças foram tratadas pelo modelo de oposições múltiplas. Seis das dez crianças também foram tratadas com pares mínimos e cinco receberam o tratamento pelo Naturalistic Speech Intelligibility $(\mathrm{NSI})^{42}$ que sugere a intervenção direta de um som-alvo em atividades naturalísticas, ou seja, do dia-a-dia, onde são dadas oportunidades que o som ocorra.

A maioria das crianças dessa pesquisa necessitou mais de um modelo de intervenção para reestruturar seu sistema fonológico com exceção apenas de duas crianças que foram tratadas apenas com o modelo de oposições múltiplas. Observou-se que as crianças com desvio fonológico profundo necessitaram de mais diversidade de intervenção do que as crianças com desvio moderado e grave.

A estrutura de terapia utilizada inicialmente era o modelo de oposições múltiplas com o máximo de pares de palavras contrastivos. À medida que a criança progredia o modelo de intervenção era mudado para incluir pares mínimos que envolviam distinções mínimas de traços. Finalmente, a terapia passava para o nível conversacional, usando o modelo NSI se a criança ainda não tivesse generalizado. Essa incorporação de modelos diferentes de intervenção, segundo a autora, pode facilitar o aprendizado fonológico.

Os resultados dessa pesquisa mostraram que todas as crianças reestruturaram seu sistema fonológico após a terapia e sugerem ainda que a mudança fonológica pode estar relacionada a três fatores: (1) ao modelo de intervenção, (2) a estrutura da intervenção e (3) ao uso de fatores sistêmicos nos alvos selecionados.

Com este estudo a autora concluiu que, estudar casos clínicos que examinem diferentes variáveis envolvendo a intervenção fonológica deve estabelecer estruturas clínicas para futuras investigações e ainda procedimentos ligados à pesquisa com a clínica científica na ciência e prática da intervenção fonológica.

O modelo de oposições múltiplas é uma variação do modelo de pares mínimos/oposições máximas, e é designado especificamente para o tratamento dos múltiplos fonemas substituídos e não da variabilidade. $O$ tratamento promove um ajustamento sistemático de um grande grupo contrastivo de sons, não apenas uma série de pares mínimos ${ }^{43}$.

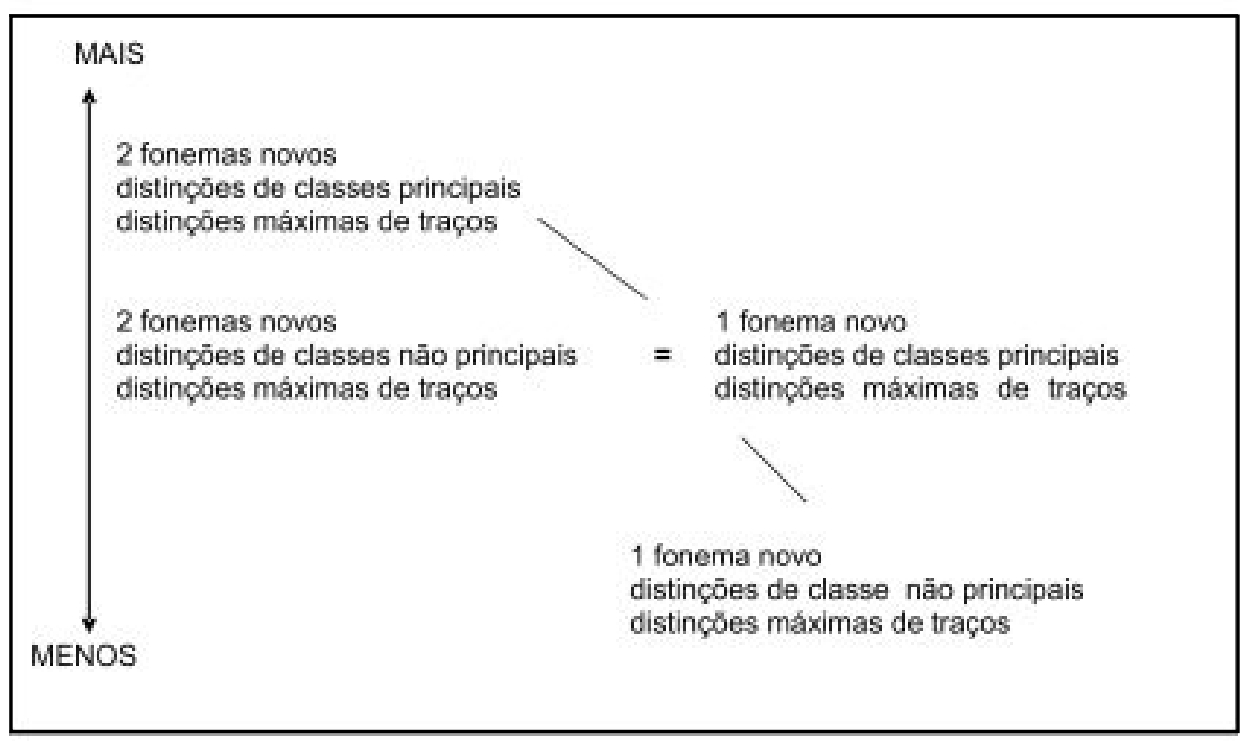

Figura 1 - Hierarquia dos formatos de tratamento com pares mínimos e as previsões de mudanças fonológicas nos sistemas fonológicos com desvios

\section{DISCUSSÃO}

Para avaliar as alterações de fala, é necessário que o terapeuta tenha conhecimento dos padrões normais de pronúncia de sua comunidade lingüística, assim como conhecer os padrões normais de desenvolvimento da fala, para realizar um diagnóstico adequado e traçar o plano terapêutico eficaz ${ }^{22}$.

A terapia com base fonológica tem como objetivo eliminar os processos existentes na fala da criança e 
reorganizar o sistema de sons com base no modelo adulto ${ }^{26}$. E tem como meta a generalização ${ }^{32}$, ou seja, não é necessário ensinar um fonema de cada vez e em cada posição para criança. Ensina-se o fonema $|z|$, por exemplo, em uma posição, e ela pode generalizar para outra posição na palavra, para outros fonemas, etc.

A estratégia do tratamento com pares mínimos/ oposições máximas e oposições múltiplas é conscientizar o sujeito de que suas produções não estão sendo efetivas, na medida em que as alterações na fala levam à produção de uma palavra não desejada, idêntica a outro item lexical presente no sistema de sua língua ${ }^{16,37}$.

O objetivo da utilização de contrastes de pares mínimos/oposições máximas no tratamento de crianças com desvios fonológicos é fazê-las distinguir pares de sílabas ou palavras (através de discriminação, imitação, e/ou produção espontânea) que são únicos em suas características ou dimensões. Através do tratamento com pares mínimos, uma criança é ensinada que diferentes sons assinalam diferentes significados. $O$ tratamento que utiliza contraste de pares mínimos reduz a homonímia na produção da criança pelo contraste realizado entre os sonsalvo errados ou substituídos ${ }^{35}$.

O Modelo de Oposições Múltiplas permite à criança fazer conexões sobre suas estratégias fonológicas com o que precisa ser aprendido e ser capaz de corrigir as estratégias baseadas no confronto de novas informações fonológicas. Além disso, o modelo é único para cada criança, pois cada uma possui suas próprias estratégias ou modelos fonológicos ${ }^{4}$.

Tanto o modelo de pares mínimos/oposições máximas como o modelo de oposições múltiplas lida com homônimos através de oposições contrastivas, mas o modelo fonológico muda para uma teoria diferente, que focaliza níveis deferentes de organização fonológica. A perspectiva do modelo de Pares Mínimos, por exemplo, enfatiza o nível do som enquanto que para o de Oposições Múltiplas é importante o nível do sistema ${ }^{4}$.

A intervenção dos pares mínimos utiliza um contraste para cada alvo, enquanto que as oposições múltiplas contrastam todos os sons-alvo com o que é substituído ${ }^{4}$.

Uma pesquisa ${ }^{37}$ utilizou o Modelo de Oposições Mínimas e o Modelo de Oposições Máximas, com o objetivo de verificar qual dos formatos de tratamento conduzia a maior mudança fonológica. Verificou-se que - Modelo de Oposições Máximas, em que foram contrastados fonemas que diferiam em múltiplos traços e que apresentavam diferenças de traços de classe principal, ocasionou melhor desempenho nos fonemas tratados e no número de fonemas adquiridos que não foram tratados, quando comparado ao Modelo de Oposições Mínimas (contrastados fonemas que diferiam em poucos traços, os quais se diferenciavam somente em traços de classe não principal).

O Modelo de Pares mínimos/Oposições Máximas é mais eficaz em crianças com grau de desvio médio a moderado-grave ${ }^{41}$, enquanto que o Modelo de Oposições Múltiplas é recomendado para crianças com desvio grave principalmente ${ }^{4}$.

Um estudo ${ }^{20}$ aplicou o Modelo de Oposições Máximas Modificado ${ }^{11}$, para falantes do português, a fim de analisar e comparar as mudanças fonológicas ocorridas nos diferentes graus de gravidade do desvio fonológico em sete crianças, quatro do sexo masculino e três do feminino, com idades entre 3:10 e 6:9. concluiu-se que o Modelo de Oposições Máximas Modificado foi efetivo para as crianças estudadas, pois proporcionou mudanças fonológicas após a aplicação do modelo. Quanto aos diferentes graus de gravidade, as maiores mudanças fonológicas, (referentes às generalizações) ocorreram nos grupos com desvios médio-moderado e moderado-grave.

O modelo de oposições múltiplas ${ }^{4}$ foi proposto como um modelo alternativo utilizado no tratamento de crianças falantes do inglês com desvios fonológicos graves. Essas crianças substituem vários fonemas do sistema adulto, reduzindo-os a um único som. Conseqüentemente, a função contrastiva de muitos sons do sistema adulto está ausente, o que reduz significativamente a inteligibilidade de fala da criança. Esse modelo baseia-se na premissa de que o nível sistêmico da organização fonológica é essencial na descrição e intervenção dos sistemas de sons desviantes.

\section{CONCLUSÃO}

A escolha de um dos modelos de oposições deve ser realizada a partir de uma avaliação criteriosa do sistema fonológico da criança, levando-se em consideração a presença e ausência dos fonemas e, também, o tipo e o grau de gravidade do desvio fonológico.

Os modelos terapêuticos que utilizam abordagem contrastiva são o de Pares Mínimos/Oposições Máximas e o de Oposições Múltiplas, sendo que no primeiro, quando o tratamento é com pares mínimos, trabalha-se com o contraste de até dois traços distintivos, enquanto que, nas oposições máximas e múltiplas, são trabalhados os contrastes de dois ou mais traços distintivos.

Os Modelos de Pares Mínimos/Oposições Máximas são aplicados em crianças que apresentam desvio fonológico médio a médio-moderado, podendo, também, ser aplicados em crianças com desvio moderado-grave e grave. Enquanto que o modelo de Oposi- 
ções Múltiplas é aplicado em crianças que apresentam desvio grave.

O Modelo de Oposições Máximas Modificado trata-se de uma variação do Modelo de Pares Mínimos/
Oposições Máximas e tem como diferencial a estrutura da sessão terapêutica, pois acrescenta a realização de bombardeio auditivo, a orientação aos pais e o uso de palavras-alvo com significado.

\section{ABSTRACT}

Purpose: this study aims at reviewing the literature on therapeutic patterns utilized in the phonologic therapy, emphasizing the contrastive approaches, being these the Minimal Pairs/Maximum Oppositions and Multiple Oppositions Approaches. Method: searches were accomplished throughout publications based on Medline, Scielo, Bireme and Pubmed data, books, thesis, dissertations, monographs and articles. Results: the Minimal Pairs/ Maximum Opposition Approaches involve the selection of couples of words that are distinguished by a single phoneme, and they may contrast few distinctive features (minimum pairs) or many features (maximum opposition). These patterns are recommended for children suffering from mild to mild-moderate disorder. In the Multiple Opposition Approach, there are words that also differ in just one phoneme, however, these contrast diverse features due to the selection of many phonemes. This pattern is recommended for severe disorder, because, in general, the children replace many phonemes of the adult system by just one sound. Conclusion: to choose the appropriate pattern, you must take into account the presence and the lack of phonemes in the sound system of the child, the kind and the severity level of the phonological disorder. Moreover, the selection of the appropriate therapeutic pattern can help to obtain future generalizations.

KEYWORDS: Articulation Disorders; Speech; Speech Therapy

\section{REFERÊNCIAS}

1.Grunwell P. The nature of phonological disability in children. London: Academic Press; 1981.

2.Grunwell P. Os desvios fonológicos numa perspectiva lingüística. In: Yavas M. Desvios fonológicos em crianças: teoria, pesquisa e tratamento. Porto Alegre: Mercado Aberto; 1990. p. 53-77.

3.Lowe RJ, Weitz JM. Intervenção. In: Lowe RJ. Fonologia: avaliação e intervenção: aplicações na patologia da fala. Porto Alegre: Artes Médicas; 1996. 4.Williams AL. Multiple oppositions: theoretical foundations for an alternative contrastive intervention approach. Am J Speech-Lang Pathol. 2000; 9:282-8. 5.Williams LA. Multiple oppositions: case studies of variables in phonological intervention. Am J SpeechLang Pathol. 2000; 9:289-99.

6. Mota HB, Pereira LF. A generalização na terapia dos desvios fonológicos: experiência com duas crianças. Pró-Fono. 2001; 13:141-6.

7.Barlow JA, Gierut JA. Minimal pair approaches to phonological remediation. Semin Speech Lang. 2002; 23(1):57-68.

8.Silva KC, Ramos APF, Wippell MLM. O modelo de oposições máximas como instrumental terapêutico em três casos de desvios fonológicos. J Bras Fonoaudiol. 2002; 3:292-7.

9.Pagan LO, Wertzner HF. Intervenção do distúrbio fonológico por meio dos pares mínimos com oposição máxima. Pró-Fono. 2002; 14:313-24.

10. Barberena LS, Keske-Soares M, Mota HB. Generalização no tratamento com o/R/ em um caso de desvio fonológico médio-moderado. Rev Soc Bras Fonoaudiol. 2004; 9:229-36.

11. Bagetti T, Mota HB, Keske-Soares M. Modelo de oposições máximas modificado: uma proposta de tratamento para o desvio fonológico. Rev Soc Bras Fonoaudiol. 2005; 10:36-41.

12. Lowe RJ. Fonologia: avaliação e intervenção: aplicações na patologia da fala. Porto Alegre: Artes Médicas; 1996.

13. Dean E, Howell J. Developing linguistic awareness: a theoretically based approach to phonological disorders. Br J Disord Commun. 1986; 21(2):223-38. 14. Tyler AA, Edwards ML, Saxman JH. Clinical application of two phonological treatment procedures. J Speech Hear Disord. 1987; 52(4):393-409.

15. Tyler AA, Figurski GR. Phonetic inventory changes after treating distinctions along an implicational hierarchy. Clin Ling Phonet. 1994; 8:91-107.

16. Weiner $F$. Treatment of phonological disability using the method of meaningful minimal contrast: two case studies. J Speech Hear Disord. 1981; 46(1):97-103. 17. Gierut JA. The conditions and course of clinicallyinduced phonological change. J Speech Hear Res. 1992; 35(5):1049-63. 
18. Pereira LF. Tratamento fonológico baseado nos contrastes de oposições máximas [mestrado]. Santa Maria (RS): Universidade Federal de Santa Maria; 1999. 19. Bagetti T. Estudo da generalização em sujeitos com desvio fonológico médio-moderado submetidos ao Modelo Terapêutico de Oposições Máximas [monografia]. Santa Maria (RS): Universidade Federal de Santa Maria; 2003.

20. Bagetti T. Mudanças fonológicas em sujeitos com diferentes graus de severidade do desvio fonológico tratados pelo Modelo de Oposições Máximas Modificado [mestrado]. Santa Maria (RS): Universidade Federal de Santa Maria; 2005.

21. Leonard BL. Deficiência fonológica. In: Fletcher P, McWhinney B. Compêndio da linguagem da criança. Porto Alegre: Artes Médicas; 1997.

22. Mota HB. Terapia fonoaudiológica para os desvios fonológicos. Rio de Janeiro: Revinter; 2001. 109 p. 23. Lamprecht RR. Aquisição fonológica do português. Porto Alegre: Artmed; 2004.

24. Hodson BW, Paden EP. Targeting intelligible speech: a phonological approach to remediation. 2. ed. San Diego: College-Hill Press; 1991.

25. Grunwell P. Developmental phonology disability: order in disorder. In: Hodson BW, Edwards ML. Perspectives in applied phonology. Gaithersburg: Aspen Publishers; 1997. p. 61-103.

26. Ingram D. The categorization of phonological impairment. In.: Hodson BW, Edwards ML. Perspectives in applied phonology. Gaithersburg: Aspen Publishers; 1997. p. 19-41.

27. Keske-Soares M. Terapia fonoaudiológica fundamentada na hierarquia implicacional dos traços distintivos aplicada em crianças com desvios fonológicos [doutorado]. Porto Alegre (RS): Pontifícia Universidade Católica do Rio Grande do Sul; 2001.

28. Wertzner HF. O distúrbio fonológico em crianças falantes do português: descrição e medidas de severidade [livre docência]. São Paulo (SP): Universidade Federal de São Paulo; 2002.

29. Blanco APF. Caracterização do grau de severidade do desvio fonológico a partir de índices de substituição e omissão [monografia]. Santa Maria (RS): Universidade Federal de Santa Maria; 2002.

30. Shriberg LD, Kwiatkowski J. Phonological disorders I: a diagnostic classification system. J Speech Hear Disord. 1982; 47(3):226-41.

31. Edwards ML. Clinical forum: phonological assessment and treatment in support of phonological processes. Lang Speech Hear Serv Sch. 1992; 23:233-40.

32. Elbert M, Gierut JA. Handbook of clinical phonology. London: Taylor \& Francis; 1986.

33. Ardenghi LA. A Terapia Metaphon em casos de desvios fonológicos [mestrado]. Santa Maria (RS): Universidade Federal de Santa Maria; 2004.

34. Mota HB. Uma abordagem terapêutica baseada nos processos fonológicos no tratamento de crianças com desvios fonológicos [mestrado]. Porto Alegre (RS): Pontifícia Universidade Católica do Rio Grande do Sul; 1990.

35. Gierut JA. Maximal opposition approach to phonological treatment. J Speech Hear Disord. 1989; 54(1):9-19.

36. Cooper R. The method of meaningful minimal contrasts in functional articulation problems. J Speech Hear Assoc Viginia. 1968; 10:17-22.

37. Gierut JA. Differential learning of phonological oppositions. J Speech Hear Disord. 1990; 33(3):540-9. 38. Chomsky N, Halle M. The sound pattern of english. New York: Harper and Row; 1968.

39. Mota HB, Pereira LF. Tratamento fonológico nos contrastes de oposições máximas. Pró-fono. 2002; 14:165-74.

40. Mota HB, Bagetti T, Keske-Soares M, Pereira LF. A generalização baseada nas relações implicacionais em sujeitos submetidos à terapia fonológica. Pró-Fono. 2005; 17:99-110.

41. Ceron MI. Generalização em três modelos de terapia fonológica aplicados em crianças com diferentes graus de severidade do desvio fonológico [monografia]. Santa Maria (RS): Universidade Federal de Santa Maria; 2007.

42. Camarata SM. The application of naturalistic conversation training to speech production in children with speech disabilities. J Appl Behav Anal. 1993; 26(2):173-82.

43. Williams L. On "minimal pair approaches to phonological remediation," (Semin speech lang 2002; 23:57-68). Semin Speech Lang. 2003; 24(3):257-8.

RECEBIDO EM: 21/03/2007

ACEITO EM: 10/09/2007

Endereço para correspondência:

Rua Coronel Scherer, 09

São Pedro do Sul - RS

CEP: 97400-000

Tel: (55) 32761471

E-mail:karinap_fono@yahoo.com.br 